\title{
Prediction of desmoglein-3 peptides reveals multiple shared T-cell epitopes in HLA DR4- and DR6- associated Pemphigus vulgaris
} Joo Chuan Tong 1,2 , Tin Wee Tan ${ }^{1}$, Animesh A Sinha*3 and Shoba Ranganathan*4,1

Address: ${ }^{1}$ Department of Biochemistry, Yong Loo Lin School of Medicine, National University of Singapore, 8 Medical Drive, Singapore 117597, Singapore, ${ }^{2}$ Institute for Infocomm Research, 21 Heng Mui Keng Terrace, Singapore 119613, Singapore, ${ }^{3}$ Center for Investigative Dermatology, Division of Dermatology and Cutaneous Sciences, College of Human Medicine, Michigan State University, 4120 Biomedical and Physical Sciences Building, East Lansing, MI 48824, USA and ${ }^{4}$ Department of Chemistry and Biomolecular Sciences \& Biotechnology Research Institute, Macquarie University, Sydney NSW 2109, Australia

Email: Joo Chuan Tong - jctong@i2r.a-star.edu.sg; Tin Wee Tan - tinwee@bic.nus.edu.sg; Animesh A Sinha* - asinha@msu.edu ; Shoba Ranganathan* - srangana@els.mq.edu.au

* Corresponding authors

from International Conference in Bioinformatics - InCoB2006

New Dehli, India. 18-20 December 2006

Published: 18 December 2006

BMC Bioinformatics 2006, 7(Suppl 5):S7 doi:10.1186/1471-2105-7-S5-S7

(C) 2006 Tong et al; licensee BioMed Central Ltd

This is an open access article distributed under the terms of the Creative Commons Attribution License (http://creativecommons.org/licenses/by/2.0), which permits unrestricted use, distribution, and reproduction in any medium, provided the original work is properly cited.

\begin{abstract}
Background: Pemphigus vulgaris (PV) is a severe autoimmune blistering skin disorder that is strongly associated with major histocompatibility complex class II alleles DRBI*0402 and $\mathrm{DQBI} * 0503$. The target antigen of PV, desmoglein 3 (Dsg3), is crucial for initiating T-cell response in early disease. Although a number of T-cell specificities within Dsg3 have been reported, the number is limited and the role of T-cells in the pathogenesis of PV remains poorly understood. We report here a structure-based model for the prediction of peptide binding to DRBI*0402 and $\mathrm{DQBI} * 0503$. The scoring functions were rigorously trained, tested and validated using experimentally verified peptide sequences.

Results: High predictivity is obtained for both DRBI*0402 $\left(r^{2}=0.90, s=1.20 \mathrm{~kJ} / \mathrm{mol}, q^{2}=0.82\right.$, $\left.s_{\text {press }}=1.61 \mathrm{~kJ} / \mathrm{mol}\right)$ and DQBI*0503 $\left(r^{2}=0.95, s=1.20 \mathrm{~kJ} / \mathrm{mol}, q^{2}=0.75, s_{\text {press }}=2.15 \mathrm{~kJ} / \mathrm{mol}\right)$ models, compared to experimental data. We investigated the binding patterns of Dsg3 peptides and illustrate the existence of multiple immunodominant epitopes that may be responsible for both disease initiation and propagation in PV. Further analysis reveals that DRBI*0402 and DQBI*0503 may share similar specificities by binding peptides at different binding registers, thus providing a molecular mechanism for the dual HLA association observed in PV.

Conclusion: Collectively, the results of this study provide interesting new insights into the pathology of PV. This is the first report illustrating high-level of cross-reactivity between both PVimplicated alleles, DRBI*0402 and DQBI*0503, as well as the existence of a potentially large number of T-cell epitopes throughout the entire Dsg3 extracellular domain (ECD) and transmembrane region. Our results reveal that DR4 and DR6 PV may initiate in the ECD and transmembrane region respectively, with implications for immunotherapeutic strategies for the treatment of this autoimmune disease.
\end{abstract}




\section{Background}

Pemphigus vulgaris (PV) is a severe autoimmune blistering skin disorder due to loss of integrity of normal intercellular attachments within the epidermis and mucosal epithelium. Strong association of PV to the major histocompatibility complex (MHC) class II alleles DRB $1 * 0402$ and DQB1 0503 have been reported in the literature [16] with over $95 \%$ of PV patients possessing one or both of these alleles $[7,8]$. The molecular basis and immunological consequences of this dual HLA association has thus far not been elucidated. The target antigen of PV, desmoglein (Dsg) 3, is a $130-\mathrm{kDa}$ transmembrane glycoprotein that belongs to the cadherin superfamily of cell adhesion molecules [9]. In the early stage of disease, patients demonstrate autoimmunity only to Dsg3 and develop mucosal blisters; while at the later stage, patients exhibit non-crossreactive immunity to both Dsg3 and Dsg1 [10]. Despite several reports of T-cell specificities within Dsg3 [11-16], the number is limited and the role of T-cells in the pathogenesis of PV remains poorly understood.

Bioinformatic tools are now commonly used in facilitating T-cell epitope discovery [17-19]. Computational methods for predicting MHC-binding peptides include procedures based on sequence motifs $[13,16]$, quantitative matrices [20-22], decision trees [23,24], artificial neural networks [25,26], hidden Markov models [27] and support vector machines [28-30]. However, despite recent advances in sequence-based predictive techniques, effective models for DRB $1 * 0402$ and DQB $1 * 0503$ have been lacking, mainly due to the paucity of sufficient peptides as training data [31] as well as register-shifts (or positional differences between the core nonameric peptide in the binding groove) [31] and polymorphisms in the binding registers (A.A. Sinha, unpublished results). To date, few prediction techniques for MHC class II molecules have been developed using three-dimensional models as the dual issues of model quality and discrimination technique must be addressed [32].

Our strategy for prediction of T-cell epitopes involves the three-dimensional modeling of peptide/MHC complexes using a hybrid docking approach that integrates the strengths of Monte Carlo simulations and homology modeling $[31,33,34]$. In an earlier study, we have successfully discriminated disease-implicated from non-disease implicated and protective alleles in PV based on structural interaction rules [31]. A complementary scoring function has now been developed for effective identification of DRB1*0402 and DQB1*0503 epitopes. We investigated the T-cell epitope repertoire of the entire Dsg3 glycoprotein and show the existence of multiple extracellular and intracellular specificities within the Dsg3 self-antigen. Further analysis reveal that $\mathrm{DRB} 1{ }^{*} 0402$ and $\mathrm{DQB} 1{ }^{*} 0503$ share similar specificities by binding peptides at different core recognition regions. These data provide a molecular rationale for the association of PV to two distinct HLA molecules and impact our understanding of the mechanism of HLA mediated control of disease.

\section{Results and Discussion DRBI*0402 predictive model}

The DRB 1*0402 model shows excellent correlation with experimental binding affinities $\left(r^{2}=0.90, s=1.20 \mathrm{~kJ} / \mathrm{mol}\right.$, $\left.q^{2}=0.82, s_{\text {press }}=1.61 \mathrm{~kJ} / \mathrm{mol}\right)$. The accuracy of the prediction model was further evaluated using (i) peptides with experimental $\mathrm{IC}_{50}$ values obtained from biochemical studies and (ii) Dsg3 peptides with T-cell proliferation values obtained from functional studies.

Three threshold binding energy values (Table 1) that define levels of specificities suitable for practical applications [34] were used to evaluate the accuracy of the DRB $1{ }^{*} 0402$ prediction model on the biochemical dataset (Test set 1) - LMH (low-, medium-, high-affinity binders; $\mathrm{A}_{\mathrm{ROC}}=0.93$ ); $\mathrm{MH}$ (medium- and high-affinity binders; $\mathrm{A}_{\mathrm{ROC}}=0.86$ ) and $\mathrm{H}$ (high-affinity binders only; $\mathrm{A}_{\mathrm{ROC}}=$ $0.81)$. The results indicate that, overall, our DRB $1 * 0402$ peptide-binding models are highly accurate $\left(A_{R O C}=0.93\right)$. $\mathrm{SP}=0.80$ offers high-sensitivity predictions, whereas $\mathrm{SP}=$ 0.95 results in very few false positives but fewer true positives. The prediction results for our model were consistent with expected binding patterns of DRB $1 * 0402$ peptides and provided a sensitivity of $70 \%(\mathrm{SP}=0.95)$ for DRB1*0402-binding peptides.

Next, the predictive performance of the DRB $1 * 0402$ model was tested on the functional dataset of 25 peptides (Test set 2) with experimental T-cell proliferation values/ responses using the decision thresholds defined above. All experimental positives (Table 2) are predicted with binding energy values of $-26.64 \mathrm{~kJ} / \mathrm{mol}$ or less (high-affinity binders; $\mathrm{SE}=0.65, \mathrm{SP}=0.80$ ). Our simulation results indicate that DRB1*0402 can bind Dsg3 96-112 at threshold $-26.64 \mathrm{~kJ} / \mathrm{mol}(\mathrm{SE}=0.65, \mathrm{SP}=0.80)$ in contrast to previous qualitative study results [31], albeit at a binding energy $(-27.09 \mathrm{~kJ} / \mathrm{mol})$ close to the threshold. Dsg3 512-526 (ranked \#10), Dsg3 78-93 (ranked \#12) and Dsg3 96-110 (ranked \#19) are predicted binders (SE = $0.65, \mathrm{SP}=0.80$ ) that did not stimulate T-cell responses in the relevant experiments $[11,13,16]$. Noteworthy, peptides Dsg3 78-93 [13] and Dsg3 96-110 [16] are fully contained within Dsg3 78-94 (ranked \#11) and Dsg3 96112 (ranked \#22), both of which are experimental true positives identified in an independent study [11]. For Dsg3 78-93, the preferred binding register identified by our models is 79-87, which is shows a 5-residue shift in the binding register from the preferred core of 82-90 for Dsg3 78-94. There is no change in the binding register observed for Dsg3 96-110 and Dsg3 96-112. It is possible 
Table I: Prediction accuracy of the DRB I*0402 peptide-binding model.

\begin{tabular}{llcc}
\hline Specificity (SP) Level & Group & Sensitivity (SE) & Binding Energy Threshold (kJ/mol) \\
\hline $\mathrm{SP}=0.80$ & LMH & 0.78 & -25.55 \\
& $\mathrm{MH}$ & 0.81 & -25.79 \\
$\mathrm{SP}=0.90$ & $\mathrm{H}$ & 0.65 & -26.64 \\
& $\mathrm{LMH}$ & 0.75 & -25.79 \\
$\mathrm{MH}$ & 0.52 & -26.94 \\
$\mathrm{SP}=0.95$ & $\mathrm{H}$ & 0.30 & -28.83 \\
& $\mathrm{LMH}$ & 0.70 & -26.94 \\
& $\mathrm{MH}$ & 0.42 & -27.72 \\
& $\mathrm{H}$ & 0.25 & -30.57 \\
\hline
\end{tabular}

Sensitivity (SE) values and binding energy thresholds for DRBI*0402 peptide-binding model at specificity (SP) levels $0.80,0.90$ and 0.95 .

that variability in the binding register (\#12) or flanking peptide residues (\#19) lead to a change in T-cell stimulation.

\section{DQB I*0503 predictive model}

The DQB $1 * 0503$ prediction model correlate well with experimental data $\left(r^{2}=0.95, s=1.20 \mathrm{~kJ} / \mathrm{mol}\right)$. The DQB1*0503 model outperforms the prediction models done by Rognan et al. (1999) on training datasets of 5 HLA-A $0204\left(r^{2}=0.85, s_{\text {press }}=2.40 \mathrm{~kJ} / \mathrm{mol}\right)$ and $37 \mathrm{H} 2-\mathrm{K}^{\mathrm{k}}$ $\left(r^{2}=0.78, s_{\text {press }}=3.16 \mathrm{~kJ} / \mathrm{mol}\right)$ peptide sequences and is consistent with our DRB ${ }^{*} 0402$ prediction model $\left(r^{2}=\right.$ $0.90, s=1.20 \mathrm{~kJ} / \mathrm{mol}, q^{2}=0.82, s_{\text {press }}=1.61 \mathrm{~kJ} / \mathrm{mol}$ ) which is trained using the same number of data points. The cross-validation coefficient $q^{2}$ and the standard error of prediction $s_{\text {press }}$ are stable, with $q^{2}=0.75$ and $s_{\text {press }}=2.15 \mathrm{~kJ} /$ mol. This iterative regression procedure validates the internal consistency of the scoring function in the current model, rendering it suitable for predictions on the test dataset obtained from functional studies.

The accuracy of the DQB1*0503 prediction model was assessed on a dataset of 6 ( 5 stimulatory and 1 non-stimulatory peptides) Dsg3 peptides with known T-cell proliferation values (Table 3). All DQB1*0503-specific Dsg3 stimulatory peptides can be effectively discriminated from the background at the prediction threshold $-26.64 \mathrm{~kJ} / \mathrm{mol}$.

\section{Disease progression in PV}

A variety of studies have demonstrated that a limited set of epitopes may be present in early disease, and intramolecular epitope spreading may occur during disease transition at the B-cell level [10]. Our data support the existence of multiple immunodominant T-cell epitopes that may be responsible for both disease initiation and propagation (Figure 1 and Figure 2). These findings are in line with T-cell proliferation data obtained from DR4 and DR6 PV patients $[11,13,15,16,31]$. Our analysis showed that the potential Dsg3 T-cell epitope repertoire is well distributed throughout all five extracellular domains (ECDs) (ECD1: Dsg3 50-158; ECD2: Dsg3 159-268; ECD3: Dsg3 269-383; ECD4: Dsg3 386-499; ECD5: Dsg3 500-615) [35]. A large number of DQB1*0503-specific Dsg3 peptides were predicted to exist within the transmembrane region (Dsg3 616-640; Figure 2). Noteworthy, 13 of these peptides were found among the top 20 predictions (Table 4), suggesting that disease initiation may begin at the transmembrane region for DR6 PV patients. Such an initiation step would not be unique to $\mathrm{PV}$ as other studies have revealed disease initiation at the transmembrane region [36,37]. For DRB $1 * 0402,90 \%$ $(18 / 20)$ of the top 20 predictions were predicted to exist within the ECDs, suggesting that disease initiation in DR4 and DR6 PV patients may be different. Although two DRB1*0402-specific intracellular peptides (Dsg3 762786 , Dsg3 786-800) have been reported $[13,16]$, and were correctly detected by our model $(\mathrm{SP}=0.80, \mathrm{SE}=$ 0.65), relatively few intracellular peptides from the region, Dsg3 641-999, are predicted by our methods. It remains to be determined what proportion of the predicted epitopes are actually generated via antigen processing events in vivo, or remain subdominant or "cryptic", such that they are not available for recognition during the initial immune response. It is likely that immune responses develop against secondary epitopes at later stages of disease progression, as a result of intramolecular epitope spreading, and it will be important to delineate the cascade of disease relevant epitopes in temporal sequence in future work.

\section{DRBI*0402 and DQB I*0503 cross reactivity}

An in-depth analysis was performed to investigate the extent of overlap in the Dsg3 peptide-binding repertoires of DRB1*0402 and DQB1*0503. A panel of $93615 \mathrm{mer}$ Dsg3 sequences were generated using an overlapping sliding window of size 15 across the entire Dsg3 glycoprotein and modeled into the binding grooves of both 
Table 2: Predicted binding affinities of Dsg3 peptide sequences to DRB I*0402.

\begin{tabular}{|c|c|c|c|c|}
\hline Rank & Peptide & Sequence & Predicted BE (kJ/mol) & References \\
\hline I & Dsg3 342-358 & SVKLSIAVKNKAEFHQS & -31.46 & Veldman et al. (2004) \\
\hline $2^{\#}$ & Dsg3 342-356 & SVKLSIAVKNKAEFH & -30.88 & Moesta et al. (2002) \\
\hline 3 & Dsg3 205-22I & GTPMFLLSRNTGEVRTL & -30.44 & Veldman et al. (2004) \\
\hline $4 * \#$ & Dsg3 846-860 & LDSLGPKFKKLAEIS & -30.17 & Moesta et al. (2002) \\
\hline 5 & Dsg3 380-396 & GIAFRPASKTFTVQKGI & -29.97 & Riechers et al. (1999) \\
\hline 6@ & Dsg3 67-8I & RNPIAKITSDYQATQ & -29.94 & Moesta et al. (2002) \\
\hline 7 & Dsg3 786-800 & MNFLDSYFSQKAFAC & -29.78 & Moesta et al. (2002) \\
\hline 8 & Dsg3 190-204 & LNSKIAFKIVSQEPA & -29.74 & Wucherpfennig et al. (1995) \\
\hline 9 & Dsg3 189-205 & HLNSKIAFKIVSQEPAG & -29.24 & Veldman et al. (2004) \\
\hline $10^{*}$ & Dsg3 512-526 & SARTLNNRYTGPYTF & -29.21 & Wucherpfennig et al. (1995) \\
\hline 11 & Dsg3 78-94 & QATQKITYRISGVGIDQ & -28.37 & Veldman et al. (2004) \\
\hline $12^{*}$ & Dsg3 78-93 & QATQKITYRISGVGID & -28.30 & Wucherpfennig et al. (1995) \\
\hline 13 & Dsg3 $191-205$ & NSKIAFKIVSQEPAG & -28.16 & Moesta et al. (2002) \\
\hline 14 & Dsg3 206-220 & TPMFLLSRNTGEVRT & -28.02 & Wucherpfennig et al. (1995) \\
\hline 15 & Dsg3 210-226 & LLSRNTGEVRTLTNSL & -27.98 & Veldman et al. (2004) \\
\hline 16 & Dsg3 25I-265 & CECNIKVKDVNDNFP & -27.88 & Wucherpfennig et al. (1995) \\
\hline 17 & Dsg3 250-266 & QCECNIKVKDVNDNFPM & -27.68 & Veldman et al. (2004) \\
\hline 18 & Dsg3 483-499 & VRVPDFNDNCPTAVLEK & -27.48 & Veldman et al. (2004) \\
\hline 19*@ & Dsg3 96-110 & PFGIFVVDKNTGDIN & -27.47 & Moesta et al. (2002) \\
\hline 20 & Dsg3 762-776 & QSGTMRTRHSTGGTN & -27.28 & Wucherpfennig et al. (1995) \\
\hline 21 & Dsg3 16I-177 & IFMGEIEENSASNSLVM & -27.09 & Hertl et al. (1998) \\
\hline 22 & Dsg3 96-112 & PFGIFVVDKNTGDINIT & -27.09 & Veldman et al. (2004) \\
\hline 23@ & Dsg3 963-977 & ERVICPISSVPGNLA & -26.87 & Moesta et al. (2002) \\
\hline $24^{*}$ & Dsg3 97-111 & FGIFVVDKNTGDINI & -26.58 & Wucherpfennig et al. (1995) \\
\hline 25@ & Dsg3 810-824 & NDCLLIYDNEGADAT & -24.00 & Moesta et al. (2002) \\
\hline
\end{tabular}

Predicted binding affinities of Dsg3 peptide sequences to DRBI*0402. '\#', '@', '*' indicate binding, non-binding and non-immunostimulatory in the relevant experiments respectively.

DRB1*0402 and DQB1*0503 (explained in detail in Peptide docking).

Both the DRB1*0402 and DQB1*0503 alleles are particularly efficient in binding Dsg3-derived peptides. Furthermore, a significant level of cross-reactivity was observed between DRB $1 * 0402$ and DQB $1 * 0503$. Of the 936 overlapping 15mer peptides generated from the entire Dsg3 glycoprotein investigated in this study, 539 (57\%) were predicted high-affinity binders to both alleles at threshold $-26.64 \mathrm{~kJ} / \mathrm{mol}$. The computer simulation results are shown in Figures 1 and 2. Noteworthy, three previously defined immunoreactive segments of the Dsg3 extracellular domains (Dsg3 145-192, 240-303 and 570-614) [38] were also predicted by our models at this specific threshold. Among the known Dsg3 peptides (Tables 2 and 3), only 2 (Dsg3 191-205 and 762-776) were predicted to bind DRB $1 * 0402$ alone, consistent with our earlier qualitative results from structural studies alone [31]. 18 (Dsg3 78-94, 96-112, 161-177, 189-205, 190-204, 205-221, 206-220, 210-226, 250-266, 251-265, 342-356, 342$358,376-392,380-396,483-499,786-800,810-824$, 963-977) were predicted to bind to both DRB $1^{*} 0402$ and DQB1*0503. These observations are of particular interest in that both $\mathrm{DRB} 1{ }^{*} 0402$ and $\mathrm{DQB} 1{ }^{*} 0503$ are strongly linked to PV $[39,40]$, indicating that common or overlapping dominant epitopes may be responsible for inducing disease in DR4 and DR6 patients respectively.

Table 3: Predicted binding affinities of Dsg3 peptide sequences to DQB I*0503.

\begin{tabular}{lllll}
\hline Rank & \multicolumn{1}{c}{ Peptide } & \multicolumn{1}{c}{ Sequence } & Predicted BE (kJ/mol) & References \\
\hline I & Dsg3 206-220 & TPMFLLSRNTGEVRT & -30.53 & Wucherpfennig et al. (1995) \\
2 & Dsg3 189-205 & HLNSKIAFKIVSQEPAG & -29.10 & Veldman et al. (2004) \\
3 & Dsg3 78-94 & QATQKITYRISGVGIDQ & -27.14 & Veldman et al. (2004) \\
4 & Dsg3 190-204 & LNSKIAFKIVSQEPA & -26.88 & Wucherpfennig et al. (1995) \\
5 & Dsg3 25I-265 & CECNIKVKDVNDNFP & -26.65 & Wucherpfennig et al. (1995) \\
6* & Dsg3 762-786 & QSGTMRTRHSTGGTNKDYADGAISM & -22.42 & Wucherpfennig et al. (1995) \\
\hline
\end{tabular}

Predicted binding affinities of $\mathrm{Dsg} 3$ peptide sequences to $\mathrm{DQB} \mid * 0503$. '*' indicates non-immunostimulatory in the relevant experiments. 


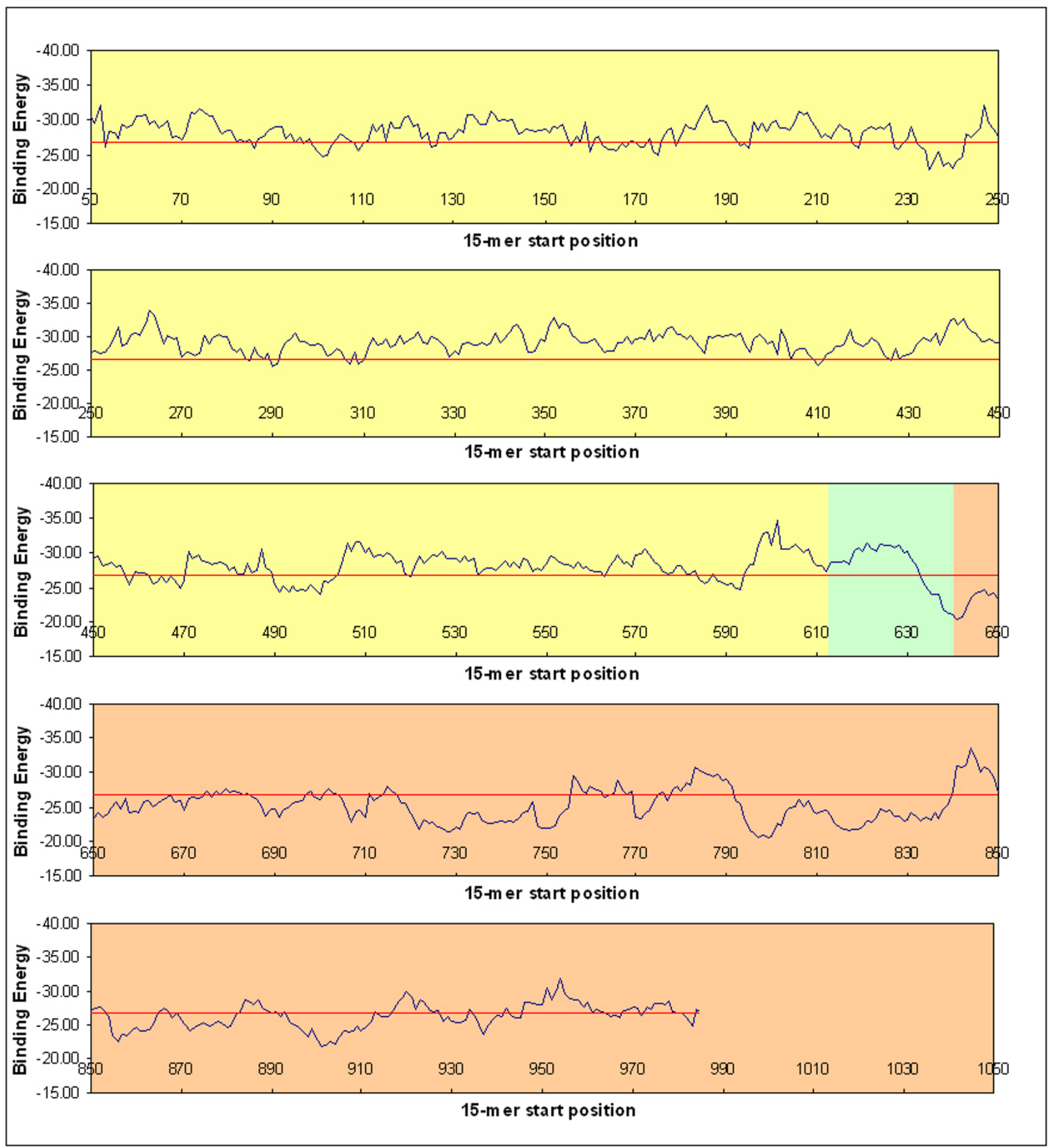

\section{Figure I}

Predicted DRB I*0402-specific T-cell epitope repertoire within Dsg3. Location of predicted DRBI*0402-specific Tcell epitopes along the Dsg3 glycoprotein - extracellular domain (colored yellow; Dsg3 50-6I5), transmembrane region (colored green; Dsg3 616-640), and intracellular domain (colored pink; Dsg3 64I-999). The red line indicates decision threshold $-26.64 \mathrm{~kJ} / \mathrm{mol}$ for high-affinity binders $(S P=0.80, S E=0.65)$. 


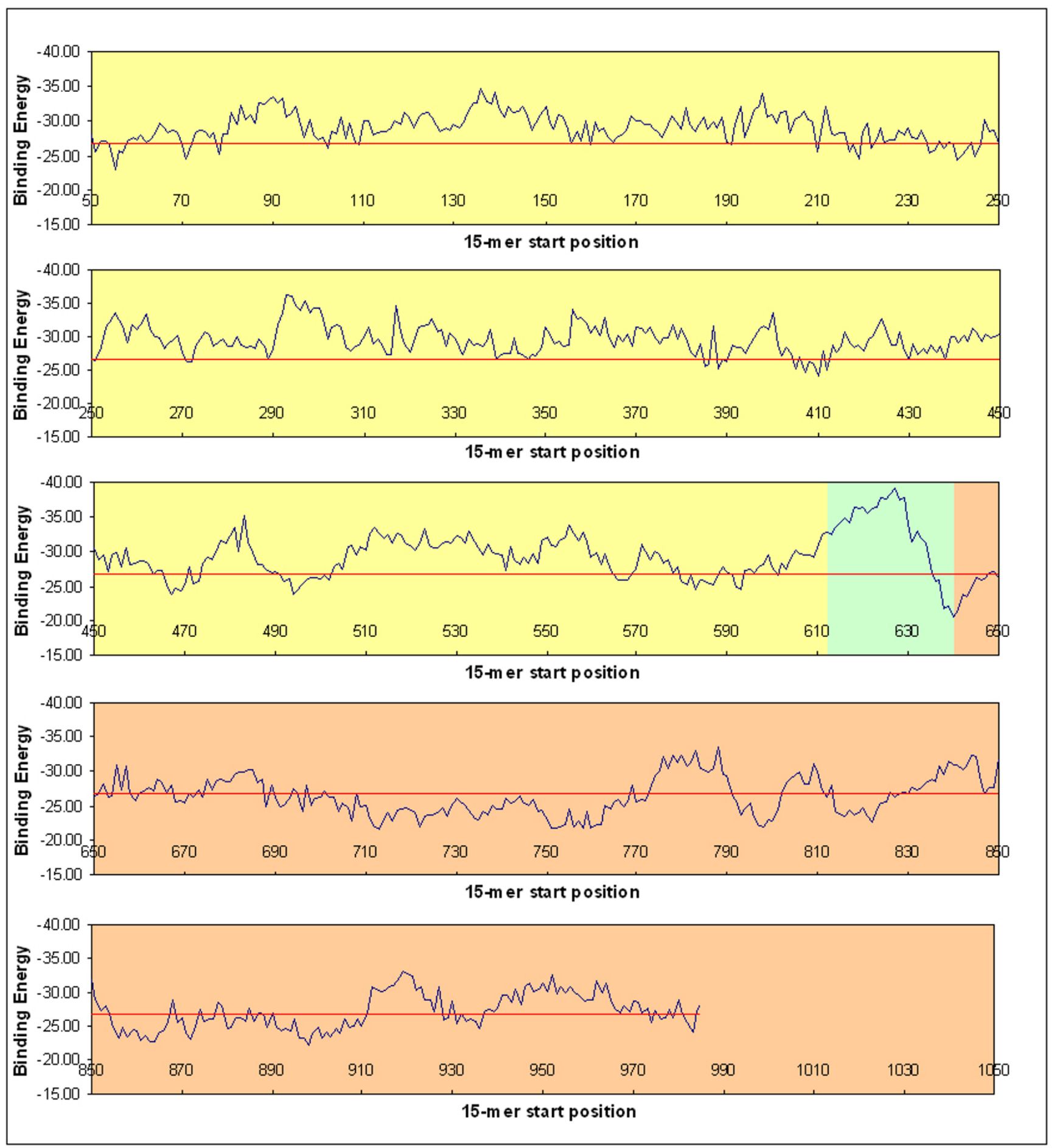

\section{Figure 2}

Predicted DQBI*0503-specific T-cell epitope repertoire within Dsg3. Location of predicted DQBI*0503-specific T-cell epitopes along the Dsg3 glycoprotein - extracellular domain (colored yellow; Dsg3 50-6I5), transmembrane region (colored green; Dsg3 6I6-640), and intracellular domain (colored pink; Dsg3 64l-999). The red line indicates decision threshold -26.64 $\mathrm{kJ} / \mathrm{mol}$ for high-affinity binders (SP $=0.80, \mathrm{SE}=0.65$ ). 
Table 4: Top 20 predicted Dsg3 peptide sequences binding to DQB I*0503 and DRB I*0402.

\begin{tabular}{|c|c|c|c|c|c|c|}
\hline \multirow[t]{2}{*}{ Rank } & \multicolumn{3}{|c|}{ DQB I *0503 } & \multicolumn{3}{|c|}{ DRB I *0402 } \\
\hline & Peptide & Sequence & Predicted B.E. (kJ/mol) & Peptide & Sequence & Predicted B.E. (kJ/mol) \\
\hline 1 & Dsg3 627-64I & GLLLLLLAPLLLLTC & -39.23 & Dsg3 60I-6I5 & TTSPGTRYGRPHSGR & -34.64 \\
\hline 2 & Dsg3 626-640 & LGLLLLLLAPLLLLT & -38.43 & Dsg3 263-277 & NFPMFRDSQYSARIE & -33.86 \\
\hline 3 & Dsg3 624-638 & LLLGLLLLLLAPLLL & -37.87 & Dsg3 844-858 & SFLDSLGPKFKKLAE & -33.46 \\
\hline 4 & Dsg3 629-643 & LLLLLAPLLLLTCDC & -37.82 & Dsg3 264-278 & FPMFRDSQYSARIEE & -33.22 \\
\hline 5 & Dsg3 625-639 & LLGLLLLLLAPLLLL & -37.65 & Dsg3 599-613 & YPTTSPGTRYGRPHS & -33.10 \\
\hline 6 & Dsg3 628-642 & LLLLLLAPLLLLTCD & -37.47 & Dsg3 352-366 & KAEFHQSVISRYRVQ & -32.88 \\
\hline 7 & Dsg3 620-634 & AIGLLLLGLLLLLLA & -36.48 & Dsg3 598-612 & SYPTTSPGTRYGRPH & -32.76 \\
\hline 8 & Dsg3 623-637 & LLLLGLLLLLLAPLL & -36.44 & Dsg3 442-456 & AEIKFVKNMNRDSTF & -32.60 \\
\hline 9 & Dsg3 618-632 & PAAIGLLLLGLLLLL & -36.36 & Dsg3 440-454 & KTAEIKFVKNMNRDS & -32.56 \\
\hline 10 & Dsg3 293-307 & LDEEYTDNWLAVYFF & -36.28 & Dsg3 439-453 & SKTAEIKFVKNMNRD & -32.36 \\
\hline 11 & Dsg3 622-636 & GLLLLGLLLLLLAPL & -36.23 & Dsg3 52-66 & VKFAKPCREGEDNSK & -32.13 \\
\hline 12 & Dsg3 619-633 & AAIGLLLLGLLLLLL & -36.20 & Dsg3 186-200 & EPNHLNSKIAFKIVS & -32.10 \\
\hline 13 & Dsg3 294-308 & DEEYTDNWLAVYFFT & -36.15 & Dsg3 354-368 & EFHQSVISRYRVQST & -32.10 \\
\hline 14 & Dsg3 62I-635 & IGLLLLGLLLLLLAP & -35.67 & Dsg3 247-26I & LSTQCECNIKVKDVN & -32.08 \\
\hline 15 & Dsg3 297-3II & YTDNWLAVYFFTSGN & -35.28 & Dsg3 262-276 & DNFPMFRDSOYSARI & -32.07 \\
\hline 16 & Dsg3 483-497 & VRVPDFNDNCPTAVL & -35.17 & Dsg3 845-859 & FLDSLGPKFKKLAEI & -32.07 \\
\hline 17 & Dsg3 616-630 & LGPAAIGLLLLGLLL & -34.86 & Dsg3 954-968 & LLTQNVIVTERVICP & -31.93 \\
\hline 18 & Dsg3 3|7-33| & EIQTDPRTNEGILKV & -34.75 & Dsg3 35I-365 & NKAEFHQSVISRYRV & -31.87 \\
\hline 19 & Dsg3 295-309 & EEYTDNWLAVYFFTS & -34.67 & Dsg3 344-358 & KLSIAVKNKAEFHQS & -31.85 \\
\hline 20 & Dsg3 136-150 & DVEKPLILTVKILDI & -34.63 & Dsg3 44I-455 & TAEIKFVKNMNRDST & -31.72 \\
\hline
\end{tabular}

The nonameric core peptide in the binding groove is shown in bold, underlined text.

DRB I*0402 and DQBI*0503 peptide binding specificities The basis for the high degree of cross-reactivities between DRB1*0402 and DQB1*0503 was subjected to further analysis. Our data support the existence of multiple binding registers within a candidate binding peptide that serve as recognition sites for DRB $1{ }^{*} 0402$ and DQB $1 * 0503$, an observation previously noted for $\mathrm{DQB} 1 * 0302$ binding peptides [34]. Of 936 Dsg3 sequences, 614 were predicted high-affinity binders with $76 \%$ displaying 2 or more registers that can be docked into the binding groove of DRB1*0402 (Figure 3). Similar results are obtained for DQB1*0503, with 673 predicted high-affinity binders and $57 \%$ exhibiting 2 or more binding registers (Figure 3 ). DRB $1 * 0402$ and DQB $1 * 0503$ predicted consensus binding sequences number 539. A striking aspect of this analysis is that DRB $1 * 0402$ and DQB $1 * 0503$ were predicted to bind a large portion of these peptides (354/539 or $66 \%$ ) at different binding registers. Noteworthy, this difference was detected in $70 \%(7 / 10)$ of Dsg3 peptides known to bind both DRB1 *0402 and DQB1*0503 (Table $5)$. For example, the consensus binding peptide Dsg 205221 showed $\Delta \mathrm{G}$ values less than the decision threshold $26.64 \mathrm{~kJ} / \mathrm{mol}$, with the 211-219 and 212-220 registers being the preferred binding modes for DRB ${ }^{*} 0402$ and DQB $1 * 0503$ respectively. We propose that DRB $1 * 0402$ and DQB1*0503 share similar specificities by binding peptides at different binding registers.

\section{Conclusion}

Although PV was first reported by Hippocrates in 400 B.C., few T-cell specificities within Dsg3 have been identified to date [11-16], and the role of MHC and T-cells in the pathogenesis of PV still remains poorly understood. Collectively, the results of this study provide interesting new insights into the pathology of PV. This is the first report of high-level of cross-reactivity between both PVimplicated alleles, DRB $1{ }^{*} 0402$ and DQB $1{ }^{*} 0503$, as well as the existence of a potentially large number of T-cell epitopes throughout the entire Dsg3 ECD and transmembrane region. Our data strongly indicates that multiple initial epitopes may be responsible for both disease initiation and progression. In addition, our modelling results revealed that DR4 and DR6 PV may initiate at different regions of Dsg3. DR4 PV may initiate in the ECD while DR6 PV may begin at the transmembrane region. It remains to be determined what proportion of predicted DRB1*0402 and DQB1*0503 binders are capable of stimulating PV-implicated alleles and autoreactive T-cells. If experimental analysis reveals limited sets of predicted Dsg3 peptides capable of eliciting functional responses, control over autoreactivity may rest at the T-cell level, rather than the level of determinant selection by $\mathrm{MHC}$ molecules. This will have a direct impact in the design of immunotherapeutic strategies for the treatment of this autoimmune disease.

\section{Methods}

MHC sequence data were obtained from IMGT-HLA database [41]. To identify potential structural templates available in the Protein Data Bank (PDB) [42] for model building, a sequence similarity search was performed using PSI-BLAST [43] running on the servers at NCBI and the highest quality templates (with the best resolution, highest sequence similarity and minimal number of miss- 


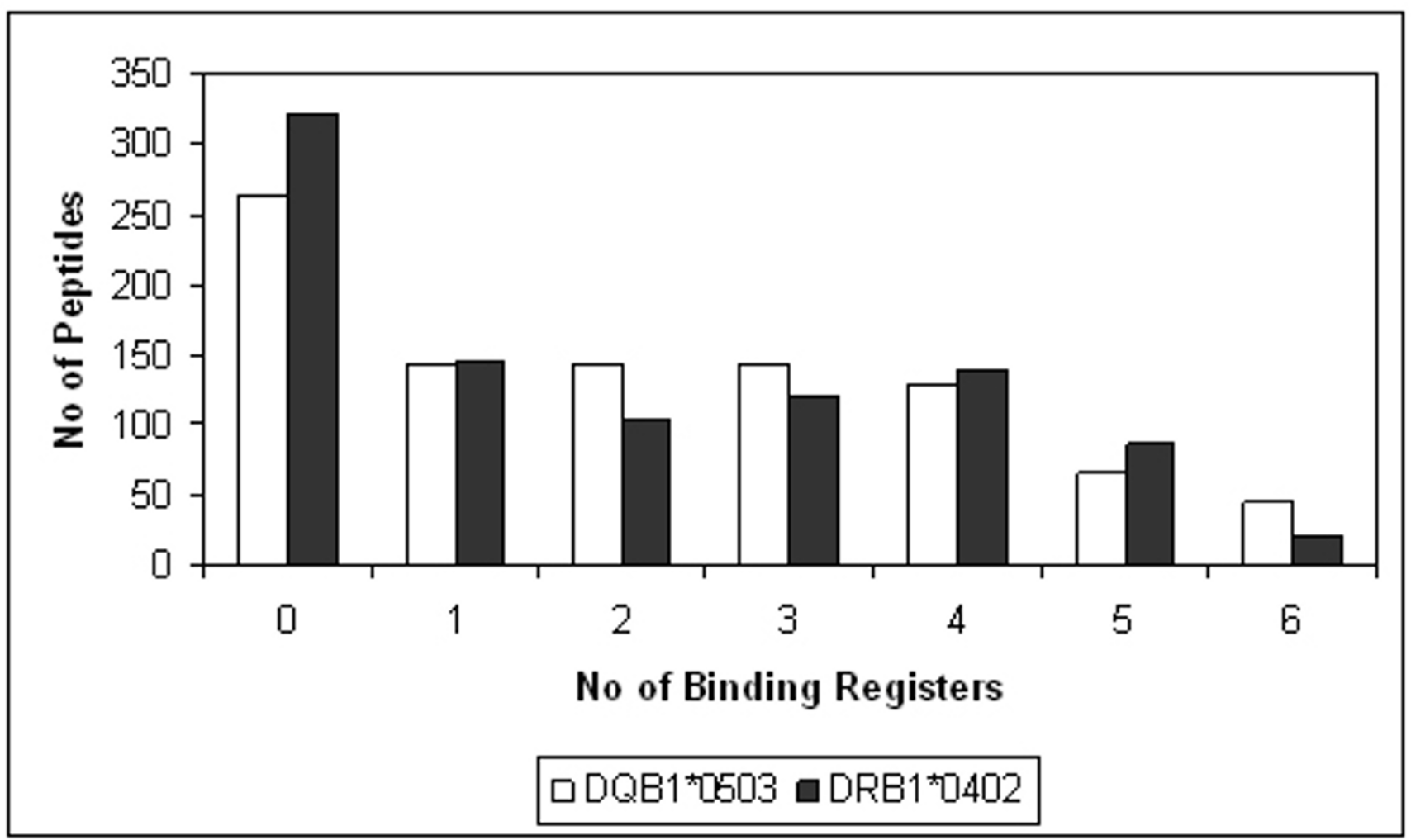

Figure 3

Predicted binding registers for Dsg3 peptides bound to DRBI*0402 and DQBI*0503. The frequency of Dsg3 peptides docked to DRBI*0402 (colored gray) and DQBI*0503 (colored white) is shown as a function of the number of predicted binding registers.

ing residues) were selected among the returned results. The crystal structures of DRB1*0401 (PDB code 1D5Z) and DQB1 *0602 (PDB code 1UVQ) were selected as templates for DRB1*0402 (97.9\% identity) and DQB1*0503 (93.0\% identity) respectively.

\section{Model building}

The program MODELLER [44] was employed for comparative modeling of both DRB $1{ }^{*} 0402$ and DQB $1 * 0503$. The models are constructed by optimally satisfying spatial constraints obtained from the alignment of the template structure with the target sequence and from the CHARMM-22 force field [45]. The structures were relaxed by conjugate gradient minimization, using the Internal Coordinate Mechanics (ICM) 3.0 package [46].

\section{Experimental binding data}

Two sets of data are used in this study: (i) peptides with experimental $\mathrm{IC}_{50}$ values from biochemical studies and (ii) peptides with experimental T-cell proliferation values/ responses from functional studies.
Dataset I (Additional file 1: Table S1) comprises 59 DRB $1 * 0402$-specific peptides derived from biochemical studies (20 high-affinity, 11 medium-affinity and 13 lowaffinity binders and 15 non-binders). Peptides are classified based on their experimental $\mathrm{IC}_{50}$ values (high-affinity binders: $\mathrm{IC}_{50} \leq 500 \mathrm{nM}$, medium-affinity binders: $500 \mathrm{nM}$ $<\mathrm{IC}_{50} \leq 1500 \mathrm{nM}$, low-affinity binders: $1500<\mathrm{IC}_{50} \leq$ $5000 \mathrm{nM}$ and non-binders: $\left.5000<\mathrm{IC}_{50}\right)$.

Dataset II (Additional file 2: Table S2) consists of 25 DRB1*0402-specific Dsg3 peptides and 14 DQB1*0503specific Dsg3 peptides with T-cell proliferation values/ responses [11-13].

\section{Peptide docking}

Overlapping 15-mer peptides are generated from the Dsg3 sequence. An overlapping sliding window of size nine is applied to each 15-mer peptide to generate all combinations of binding registers to be modeled into the binding grooves of DRB1*0402 and DQB1*0503. Docking was performed with the Empirical Conformational Energy Program for Peptides 3 force field parameters (ECEPP/3) [46] and MMFF partial charges [47] on a 4-CPU SGI Ori- 
Table 5: Preferred binding registers of experimentally determined Dsg3 peptides for PV implicated alleles.

\begin{tabular}{clllll}
\hline \multicolumn{1}{c}{ Peptide } & \multicolumn{1}{c}{ Allele } & \multicolumn{1}{c}{ Sequence } & Peptide & Allele & Sequence \\
\hline Dsg3 78-94 & DRBI*0402 & QATQKITYRISGVGIDQ & Dsg3 250-266 & DRBI*0402 & QCECNIKVKDVNDNFPM \\
& DQBI*0503 & QATQKITYRISGVGIDQ & & DQBI*0503 & QCECNIKVKDVNDNFPM \\
Dsg3 96-112 & DRBI*0402 & PFGIFVVDKNTGDINIT & Dsg3 25I-265 & DRBI*0402 & CECNIKVKDVNDNFP \\
& DQBI*0503 & PFGIFVVDKNTGDINIT & & DQBI*0503 & CECNIKVKDVNDNFP \\
Dsg3 16I-177 & DRBI*0402 & IFMGEIEENSASNSLVM & Dsg3 342-356 & DRBI*0402 & SVKLSIAVKNKAEFH \\
Dsg3 189-205 & DRBI*0402 & HLNSKIAFKIVSQEPAG & Dsg3 342-358 & DRBI*0402 & SVKLSIAVKNKAEFHQS \\
& DQBI*0503 & HLNSKIAFKIVSQEPAG & & DQBI*0503 & SVKLSIAVKNKAEFHQS \\
Dsg3 190-204 & DRBI*0402 & LNSKIAFKIVSQEPA & Dsg3 376-392 & DQBI*0503 & NVREGIAFRPASKTFTV \\
& DQBI*0503 & LNSKIAFKIVSQEPA & Dsg3 380-396 & DRBI*0402 & GIAFRPASKTFTVQKGI \\
Dsg3 19I-205 & DRBI*0402 & NSKIAFKIVSQEPAG & Dsg3 483-499 & DRBI*0402 & VRVPDFNDNCPTAVLEK \\
Dsg3 205-22I & DRBI*0402 & GTPMFLLSRNTGEVRTL & Dsg3 762-776 & DRBI*0402 & QSGTMRTRHSTGGTN \\
& DQBI*0503 & GTPMFLLSRNTGEVRTL & Dsg3 786-800 & DRBI*0402 & MNFLDSYFSQKAFAC \\
Dsg3 206-220 & DRBI*0402 & TPMFLLSRNTGEVRT & Dsg3 8I0-824 & DRBI*0402 & NDCLLIYDNEGADAT \\
& DQBI*0503 & TPMFLLSRNTGEVRT & Dsg3 963-977 & DRBI*0402 & ERVICPISSVPGNLA \\
Dsg3 210-226 & DRBI*0402 & LLSRNTGEVRTLTNSL & & &
\end{tabular}

Preferred binding registers of experimentally determined Dsg3 peptides for PV implicated alleles. Best fitting registers with lowest predicted binding energies are in bold, underlined text.

gin 3200 workstation using an extension of the protocol [33,34]: (i) pseudo-Brownian rigid body docking of peptide fragments to the ends of the binding groove, (ii) central loop closure by satisfaction of spatial constraints, (iii) refinement of the backbone and side-chain atoms of the core recognition residues and receptor contact regions within $4.00 \AA$ radius $[31,33]$ and (iv) extension of flanking peptide residues by satisfaction of spatial constraints.

\section{Empirical free energy functions}

The scoring function presented in this study is based on the free energy potential in ICM3.0 package [47]. The binding free energy function is partitioned into three terms [34] expressed by the equation:

$\Delta \mathrm{G}=\alpha \Delta \mathrm{G}_{\mathrm{H}}+\beta \Delta \mathrm{G}_{\mathrm{S}}+\gamma \Delta \mathrm{G}_{\mathrm{EL}}+C$.

$\Delta \mathrm{G}_{\mathrm{H}}$ is the hydrophobic energy computed as the product of solvent accessible surface area (determined by rolling a sphere of $1.40 \AA$ radius along the surface of the molecule) by the surface tension. $\Delta \mathrm{G}_{\mathrm{S}}$ refers to the entropic contribution from the protein side-chains computed from the maximal burial entropies for each type of amino acid and their relative accessibilities. $\Delta \mathrm{G}_{\mathrm{EL}}$ denotes the electrostatic term composed of coulombic interactions between receptor and ligand and the desolvation of partial charges transferred from an aqueous medium to a protein core environment, and is determined by the numeric solution of the Poisson equation using an implementation of the boundary element algorithm [48-50]. The constant term $C$ accounts for entropy change in the system due to the decrease of free molecular concentration and the loss of rotational/translational degrees of freedom upon binding [51].

\section{Training, testing and validation}

Two computational models are trained in this study - one model for the prediction of peptide binding to DRB1*0402 and the other for DQB1*0503.

DRB $1 * 0402$-specific peptide data derived from biochemical studies with experimental $\mathrm{IC}_{50}$ values was divided into training and test sets. The training set comprised 8 (5 binding and 3 non-binding) Dsg3 sequences with experimentally determined binding registers (from Dataset I). Two external sets of test data were used: (i) Test set 1: 51 peptides with experimental $\mathrm{IC}_{50}$ values (20 high-affinity binders, 11 medium affinity binders, 9 low affinity binders and 11 non-binders) from biochemical studies, and (ii) Test set 2: all DRB1*0402-specific Dsg3 peptides from Dataset II, with known T-cell proliferation values.

DQB $1{ }^{*} 0503$ prediction model was trained using Dsg3 peptide data from functional studies in the absence of relevant biochemical data. The training set comprised 8 (5 stimulatory and 3 non-stimulatory) sequences from Dataset II. For each peptide sequence, T-cell proliferation value [13] is mapped to a theoretical $\mathrm{IC}_{50}$ value in accordance with expected binding patterns of Dsg3 binding peptides (Sinha et al., personal communications). The performance of the prediction model was subsequently evaluated on an external set of 6 peptides with known T-cell proliferation values.

Coefficients $(\alpha, \beta, \gamma)$ and the constant term $C$ in Equation 1 were derived using standard least-square multivariate regression analyses of the training set, followed by leaveone-out analysis to assess to quality of the scoring function [52]. For each model, the entire procedure is repeated 
8 times to reduce noises in all computations, the results averaged and the observed error rate is used to estimate the expected error rate upon generalization to new data.

The optimal scoring function selected from each cross-validation analysis was further assessed using sensitivity (SE), specificity (SP) and receiver operating characteristic (ROC) analysis [34]. $\mathrm{SE}=\mathrm{TP} /(\mathrm{TP}+\mathrm{FN})$ and $\mathrm{SP}=\mathrm{TN} /$ $(\mathrm{TN}+\mathrm{FP})$, indicate percentages of correctly predicted binders and non-binders, respectively. TP (true positives) represents experimental binders with at least one predicted binding register and TN (true negatives) for experimental non-binders with no predicted binding register. FN (false negatives) denotes experimental binders predicted as non-binders and FP (false positives) represents experimental non-binders predicted as binders. The accuracy of our predictions was assessed by ROC analysis where the ROC curve is generated by plotting $\mathrm{SE}$ as a function of (1SP) for various classification thresholds. The area under the ROC curve $\left(A_{R O C}\right)$ provides a measure of overall prediction accuracy, $A_{R O C}<70 \%$ for poor, $A_{R O C}>80 \%$ for good and $A_{R O C}>90 \%$ for excellent predictions [34]. In this study, we assessed SE for three values of SP $(80 \%, 90 \%$ and $95 \%$ ) that are considered useful in practice. All regression and validation results, including correlation coefficient $\left(r^{2}\right)$, standard deviation $(s)$, cross-validation coefficient $\left(q^{2}\right)$, standard error of prediction $\left(s_{\text {press }}\right)$, SE, $\mathrm{SP}, \mathrm{A}_{\mathrm{ROC}}$, coefficients and constant terms in the scoring function, are recorded.

\section{Authors' contributions}

JCT carried out the computational simulation studies and drafted the manuscript. AAS carried out the immunoassays. TWT, AAS and SR participated in the design of the study and interpretation of data. SR developed the project and finalized the manuscript.

\section{Additional material}

\section{Additional file 1}

Table-S1

Click here for file

[http://www.biomedcentral.com/content/supplementary/14712105-7-S5-S7-S1.pdf]

\section{Additional file 2}

Table-S2

Click here for file

[http://www.biomedcentral.com/content/supplementary/14712105-7-S5-S7-S2.pdf]

\section{Acknowledgements}

This article has been published as part of BMC Bioinformatics Volume 7, Supplement 5, 2006: APBioNet - Fifth International Conference on Bioinfor- matics (InCoB2006). The full contents of the supplement are available online at http://www.biomedcentral.com/ |47| -2105/7? issue=S5.

\section{References}

I. Carcassi C, Cottoni F, Floris L, Vacca A, Mulargia M, Arras M, Boero R, La Nasa G, Ledda A, Pizzati A, Cerimele D, Contu L: HLA haplotypes and class II molecular alleles in Sardinian and Italian patients with pemphigus vulgaris. Tissue Antigens 1996, 48:662-667.

2. Delgado JC, Hameed A, Yunis JJ, Bhol K, Rojas Al, Rehman SB, Khan AA, Ahmad M, Alper CA, Ahmed AR, Yunis EJ: Pemphigus vulgaris autoantibody response is linked to HLA-DQB I*0503 in Pakistani patients. Hum Immunol 1997, 57: I 10-119.

3. Loiseau P, Lecleach L, Prost C, Lepage V, Busson M, Bastuji-Garin S, Roujeau JC, Charron D: HLA class II polymorphism contributes to specify desmoglein derived peptides in pemphigus vulgaris and pemphigus foliaceus. J Autoimmun 2000, 15:67-73.

4. Miyagawa S, Higashimine I, lida T, Yamashina Y, Fukumoto T, Shirai T: HLA-DRB I*04 and DRB I* | 4 alleles are associated with susceptibility to pemphigus among Japanese. J Invest Dermatol 1997, 109:615-618.

5. Miyagawa S, Amagai M, lida T, Yamamoto Y, Nishikawa T, Shirai T: Late development of antidesmoglein I antibodies in pemphigus vulgaris: correlation with disease progression. $\mathrm{Br} J \mathrm{Derma}$ tol |999, | 4 | : | 084- 1087.

6. Niizeki $H$, Inoko $H$, Narimatsu $H$, Takata $H$, Sonoda $A$, Tadakuma $T$, Ando A, Tsuji K, Hashimoto T, Nishikawa T: HLA class II antigens are associated with Japanese pemphigus patients. Hum Immunol 1991, 3 I:246-50.

7. Sinha AA, Brautbar C, Szafer F, Friedmann A, Tzfoni E, Todd JA, Steinman L, McDevitt HO: A newly characterized HLA DQ beta allele associated with pemphigus vulgaris. Science 1988 , 239: 1026-1029.

8. Scharf SJ, Friedmann A, Brautbar C, Szafer F, Steinman L, Horn G, Gyllensten U, Erlich HA: HLA class II allelic variation and susceptibility to pemphigus vulgaris. Proc Natl Acad Sci U S A 1988 , 85:3504-3508.

9. Amagai M, Klaus-Kovtun V, Stanley JR: Autoantibodies against a novel epithelial cadherin in pemphigus vulgaris, a disease of cell adhesion. Cell I99I, 67:869-877.

10. Salato VK, Hacker-Foegen MK, Lazarova Z, Fairley JA, Lin MS: Role of intracellular epitope spreading in pemphigus vulgaris. Clin Immunol 2005, I I 6:54-64.

II. Veldman CM, Gebhard KL, Uter W, Wassmuth R, Grötzinger J, Schultz E, Hertl M: T cell recognition of desmoglein 3 peptides in patients with Pemphigus Vulgaris and healthy individuals. J Immunol 2004, I 72:3883-3892.

12. Hertl M, Amagai M, Sundaram H, Stanley J, Ishii K, Katz SI: Recognition of desmoglein 3 by autoreactive $T$ cells in pemphigus vulgaris patients and normals. J Invest Dermatol 1998, I I 0:62-66.

13. Wucherpfennig KW, Yu B, Bhol K, Monos DS, Argyris E, Karr RW, Ahmed AR, Strominger JL: Structural basis for major histocompatibility complex (MHC)-linked susceptibility to autoimmunity: charged residues of a single MHC binding pocket confer selective presentation of self-peptides in pemphigus vulgaris. Proc Natl Acad Sci U S A 1995, 92: I I935-I I 939.

14. Riechers R, Grotzinger J, Hertl M: HLA class II restriction of autoreactive $\mathbf{T}$ cell responses in pemphigus vulgaris: review of the literature and potential applications for the development of a specific immunotherapy. Autoimmunity 1999, 30:183-196.

15. Chow S, Rizzo C, Ravitskiy L, Sinha AA: The role of T cells in cutaneous autoimmune disease. Autoimmunity 2005, 38:303-3I7.

16. Moesta M, Stevanovic S, Rammensee H-G, Lin M-S, Sinha AA: Identification of intracellular $T$ cell epitopes in pemphigus vulgaris. J Invest Dermatol 2002, I I 9:319.

17. Schirle M, Weinschenk T, Stevanovic S: Combining computer algorithms with experimental approaches permits the rapid and accurate identification of $T$ cell epitopes from defined antigens. J Immunol Methods 200I, 257:I-16.

18. Yu K, Petrovsky N, Schonbach C, Koh JY, Brusic V: Methods for prediction of peptide binding to MHC molecules: a comparative study. Mol Med 2002, 8: I37-I48.

19. Srinivasan KN, Zhang GL, Khan AM, August JT, Brusic V: Prediction of class I T-cell epitopes: evidence of presence of immuno- 
logical hot spots inside antigens. Bioinformatics 2004, 20(Suppl I):i297-i302

20. Parker KC, Bednarek MA, Coligan JE: Scheme for ranking potential HLA-A2 binding peptides based on independent binding of individual peptide side-chains. J Immunol I994, 152:163-175.

21. Davenport MP, Ho Shon IAP, Hill AVS: An empirical method for the prediction of T-cell epitopes. Immunogenetics 1995 , 42:392-397.

22. Gulukota K, Sidney J, Sette A, DeLisi C: Two complementary methods for predicting peptides binding major histocompatibility complex molecules. J Mol Biol 1997, 267:1258-1267.

23. Savoie C], Kamikawaji N, Sasazuki T, Kuhara S: Use of BONSA decision trees for the identification of potential MHC class I peptide epitope motifs. Pac Symp Biocomput 1999.

24. Segal MR, Cummings MP, Hubbard AE: Relating amino acid sequence to phenotype: analysis of peptide-binding data. Biometrics 200I, 57:632-642.

25. Brusic V, Rudy G, Harrison LC: Prediction of MHC binding peptides using artificial neural networks. In Complex Systems: Mechanism of Adaptation Edited by: Stonier RJ, Yu XS. Amsterdam: IOS Press; 1994:253-260.

26. Brusic V, Rudy G, Honeyman M, Hammer J, Harrison L: Prediction of MHC class II-binding peptides using an evolutionary algorithm and artificial neural network. Bioinformatics 1998, 14:121-130.

27. Mamitsuka $\mathrm{H}$ : Predicting peptides that bind to MHC molecules using supervised learning of hidden Markov models. Proteins 1998, 33:460-474.

28. Dönnes P, Elofsson A: Prediction of MHC class I binding peptides, using SVMHC. BMC Bioinformatics 2002, 3:25.

29. Bhasin M, Raghava GPS: SVM based method for predicting HLA DRB I*040 I binding peptides in an antigen sequence. Bioinformatics 2004, 20:42I-423.

30. Bozic I, Zhang G, Brusic V: Predictive vaccinology: optimisation of predictions using support vector machine classifiers. IDEAL 2005:375-38I.

31. Tong JC, Bramson J, Kanduc D, Chow S, Sinha AA, Ranganathan S: Modeling the bound conformation of pemphigus vulgarisassociated peptides to MHC class II DR and DQ alleles. Immunome Res 2006, 2:I.

32. Ranganathan S, Tong JC, Tan TW: Structural Immunoinformatics: Understanding MHC-peptide binding. In Advances in Experimental Medicine Series - Immunoinformatics: Opportunities and Challenges of Bridging Immunology with Computer and Information Sciences Edited by: Schoenbach C. Kluwer Plenum Press. accepted

33. Tong JC, Tan TW, Ranganathan S: Modeling the structure of bound peptide ligands to major histocompatibility complex. Protein Sci 2004, 1 3:2523-2532.

34. Tong JC, Zhang GL, Tan TW, August JT, Brusic V, Ranganathan S: Prediction of HLA-DQ3.2 $\beta$ ligands: Evidence of multiple registers in class II binding peptides. Bioinformatics 2006 22:1232-1238

35. Amagai M, Hashimoto T, Shimizu N, Nishikawa T: Absorption of pathogenic autoantibodies by the extracellular domain of pemphigus vulgaris antigen (Dsg3) produced by baculovirus. Clin Invest 1994, 94:59-67.

36. Akin C, Fumo G, Yavuz AS, Lipsky PE, Neckers L, Metcalfe DD: A novel form of mastocytosis associated with a transmembrane c-kit mutation and response to imatinib. Blood 2004, 103:3222-3225.

37. Theophilos MB, Cox DW, Mercer JF: The toxic milk mouse is a murine model of Wilson disease. Hum Mol Genet 1996, 5:1619-1624.

38. Lin MS, Swartz SJ, Lopez A, Ding X, Fernandez-Vina MA, Stastny P, Fairley JA, Diaz LA: Development and characterization of desmoglein-3 specific $T$ cells from patients with pemphigus vulgaris. J Clin Invest 1997, 99:3 I-40.

39. Lee EY, Sinha AA: T cell targeted therapy for autoimmune disease. Autoimmunity 2005, 38:577-596.

40. Sinha AA, Lopez MT, McDevitt HO: Autoimmune diseases: the failure of self-tolerance. Science 1990, 248:1380-1388.

4I. Robinson J, Waller MJ, Parham P, de Groot N, Bontrop R, Kennedy LI, Stoehr P, Marsh SG: IMGT/HLA and IMGT/MHC: sequence databases for the study of the major histocompatibility complex. Nucleic Acids Res 2003, 31:3 I I-314.
42. Berman HM, Westbrook J, Feng Z, Gilliland G, Bhat TN, Weissiq H, Shindyalov IN, Bourne PE: The Protein Data Bank. Nucleic Acids Res 2000, 28:235-242.

43. Altschul SF, Gish W, Miller W, Myers EW, Lipman DJ: Basic local alignment search tool. J Mol Biol 1990, 21 5:403-4I0.

44. Sali A, Blundell TL: Comparative protein modelling by satisfaction of spatial restraints. J Mol Biol 1993, 234:779-8I5.

45. MacKerell AD, Bashford D, Bellott M, Dunbrack RL, Evanseck JD, et al: All-atom empirical potential for molecular modeling and dynamics studies of proteins. J Phys Chem 1998, B 102:3586-36I7.

46. Abagyan R, Totrov M, Kuznetsov D: ICM - a new method for protein modeling and design: Applications to docking and structure prediction from the distorted native conformation. $J$ Comput Chem 1994, 15:488-506.

47. Abagyan RA, Totrov M: Ab initio folding of peptides by the optimal-bias Monte Carlo minimization procedure. J Comput Phys |999, | 5 |:402-42 |.

48. Zauhar RJ, Morgan RS: A new method for computing the macromolecular electric potential. J Mol Biol I985, 186:8I5-820.

49. Bharadwaj A, Windemuth A, Sridharan S, Honig B, Nicholls A: The fast multipole boundary element method for molecular electrostatics: an optimal approach for large system. J Comput Chem 1995, 16:898-910.

50. Schapira M, Totrov M, Abagyan R: Prediction of the binding energy for small molecules, peptides and proteins. J Mol Recognit 1999, I 2:177-190

5I. Janin J: Protein-protein recognition. Prog Biophys Mol Biol 1995, 64:145-166.

52. Rognan D, Lauemøller SL, Holm A, Buus S, Tschinke V: Predicting binding affinities of protein ligands from three-dimensional models: Application to Peptide Binding to Class I Major Histocompatibility Proteins. J Med Chem 1999, 42:4650-4658.

Publish with BioMed Central and every scientist can read your work free of charge

"BioMed Central will be the most significant development for disseminating the results of biomedical research in our lifetime. "

Sir Paul Nurse, Cancer Research UK

Your research papers will be:

- available free of charge to the entire biomedical community

- peer reviewed and published immediately upon acceptance

- cited in PubMed and archived on PubMed Central

- yours - you keep the copyright 